\title{
Feed tests, models helping to control BSE
}

$\mathrm{O}$ ver the course of 2 decades, UC San Francisco neurologist and Nobel laureate Stanley Prusiner proved that the infectious agent in mad cow disease and related brain-wasting syndromes was a misfolded protein called a prion. Today, UC scientists in several disciplines continue to combat prion-caused diseases (or transmissible spongiform encephalopathies), working to control them through both prevention and treatment.

Prusiner, who won the 1997 Nobel Prize for his discovery of "proteinaceous infectious particles" (prions), demonstrated that these agents engender fatal brain diseases that occur in humans, cattle, sheep, elk, deer and other animals. "By showing that these misfolded proteins could be infectious agents, he redefined the long-accepted principles of infectious disease, creating the need for a new paradigm addressing its prevention and treatment," says Donald Klingborg, associate dean of the UC Davis School of Veterinary Medicine.

Among UC scientists focusing on mad cow disease is a team at UC Davis that has developed a new cattle-feed test to help keep the disease from spreading, and another team doing molecular-level modeling that could eventually yield treatments for the disease.

Feed test. Cattle can catch BSE from feed that contains byproducts from infected cows. While banned from cattle feed, byproducts from cows and other ruminants are allowed in poultry and swine feed. That means cattle feed can be contaminated accidentally if, for example, a feed mill is not cleaned properly between producing different types of feed.

The new test is DNA-based and can detect smaller amounts of ruminant contaminants than the current antibody-based test (see page 212). "The DNA test is 10 to 100 times more sensitive," says James Cullor of the UC Davis School of Veterinary Medicine (based in Tulare), who led the team that developed the new test. However, the antibody test is faster than the DNA test: the former takes only 25 minutes while the latter can take up to 6 hours for complicated feeds, which can contain grain, fruit, silage and even rejected M\&Ms.

Now Cullor and his team are fine-tuning the DNA test, in part because the federal government wants it to work on European feed, which has smaller pieces of DNA due to processing differences. "We will keep refining the test to make it faster, better and less expensive," he says.

Prion models. Having a sensitive test for ruminant byproducts is critical because it does not take much to infect cows. BSE is caused by prions, an abnormal form of a protein found mostly on the surfaces of neurons and lymph system cells. Prions are folded incorrectly and can convert the normal proteins into more prions, which then stick together in aggregates called plaques. Much is still unknown about BSE, including why the incubation period is so long - up to 10 years in cattle and up to 30 years in people. It is also unknown how BSE crossed the species barrier to infect humans, an event first documented in the United Kingdom in 1996. While the human disease, named "variant Creutzfeldt-Jakob disease" is infectious, other diseases characterized by abnormal plaque accumulation are not. (These include Alzheimer's, Parkinson's and type II diabetes.)

UC Davis biophysicist Daniel Cox and his colleagues have developed models of prions, because these mis- 